\title{
Latent Attitude Method for Trend Analysis with Pooled Survey Data
}

\author{
Donghui Wang*1, Yu Xie ${ }^{2,3}$, and Juming Huang ${ }^{2}$ \\ ${ }^{1}$ Center for Population and Development Studies, School of Sociology and Population \\ Studies, Renmin University of China \\ ${ }^{2}$ The Paul and Marcia Wythes Center on Contemporary China, Princeton University \\ ${ }^{3}$ Center for Social Research, Peking University
}

April 28, 2021

\begin{abstract}
Millions of people are surveyed every year regarding their attitudes toward various topics. Together these surveys have produced a large corps of data that document how people think collectively toward various aspects of contemporary social life. The wealth of the attitude surveys has promoted scholars to move beyond the single-survey analysis. However, the use of survey data for studying trends in attitudes is handicapped by a measurement difficulty: different surveys have used different survey instruments to measure the same attitude and thus have generated data that strictly non-comparable. We propose the Latent Attitude Method (LAM) to address this issue. Our method borrows strength from two research traditions: (1) the latent variable method in attitude research and (2) the comparable distribution condition in survey design and evaluation. The core of this method is that, when two or more surveys overlap in a given year, we assume that the same latent attitude is measured as if two measurement scales are randomly given to two independent samples drawn from the same population. Thus, we can assume the same statistical properties for the latent attitude. In so doing, we are able to reduce the number of unknowns to be less than the number of established equations and estimate the best-fit parameters with maximum likelihood method. We demonstrate the utility of the method with simulated data, and apply the method to an empirical example of estimating America's attitude toward China from 1974 to 2019
\end{abstract}

*donghuiwangruc@gmail.com 


\section{Introduction}

Social science has long thought to understand the causes, trends, and consequences of social attitudes, studying such a wide-range of topics as attitudes toward gender roles (Cotter et al., 2011, Scott et al., 1996), marriage (Thornton, 1985), race relations (Steeh and Schuman, 1992), inequality (Osberg and Smeeding, 2006), migration (Semyonov et al., 2011), and political affairs (DiMaggio et al., 1996). The advancements of attitude research are largely facilitated by the proliferation of large-scale attitude surveys. For example, the General Social Survey that began in 1972 has generated nationally representative data over time that capture the ebbs and flows of American's attitudes toward hundreds of issues, ranging from government spending to income inequality to climate change (National Opinion Research Center, 2020). Over the past several decades, the number of attitude surveys have grown exponentially, owning to the maturation and adaptation of modern survey methodology as well as advancement of computer technologies that greatly reduced the costs in survey design, interview, data storage and analysis. To date, millions of individuals are surveyed every year by academic institutions, commercial companies, and news media organizations regarding their attitudes toward various topics. Together these surveys have produced a large corps of data that document how people think collectively toward various aspects of contemporary social life.

Despite the large size of accumulated attitude survey data over years, use of the data for studying trends in attitudes is handicapped by a measurement difficulty: different surveys have used different survey instruments to measure the same attitude and thus have generated data that strictly non-comparable. For example, individuals' attitudes toward an particular social issue can be measured as a four-point Likert scale in one survey, while a 10-point scale in another survey. There is a growing need to harmo- 
nize different attitude surveys to understand attitude changes over time (e.g. Stimson, 2018;DeJonge et al., 2016).

This need becomes particularly acute when a time series of cross-sectional surveys either begins or stops. Pooling surveys with different time series coverage allows researchers to study societal attitude changes with a much longer observation window. Furthermore, pooling different surveys in a small window of time also help triangulate research findings. Especially if the targeted population are the same across surveys, researchers can leverage different surveys one against the other, so as to track genuine changes of attitudes (e.g. Jackman, 2005).

Past studies have attempted to ameliorate this problem in two ways. The first approach is to simply collapse the ordinal response categories into binary responses that indicate respondents' approval / disapproval of the survey statements. This method is easy to implement and can yield informative results. However, not only does method result in loss of information, it also forces the researcher to choose an arbitrary cut-point to dichotomize approval versus disapproval responses.

The second approach is to find secondary metrics and convert all the original scales accordingly. The secondary metrics are usually drawn from the marginal distribution of the empirical survey response in a given year. In practice, researchers generally choose the year when two time-series surveys overlap. For example, DeJong and colleagues developed the Reference Distribution Method that addresses scale incomparability of happiness measurements across surveys (De Jonge et al., 2017). To apply the method, researchers first need to choose the marginal distribution of a survey item in a given year as the reference. Once the reference survey is chosen, researchers then estimate the coefficients of a beta distribution that best describe the chosen marginal distribution. The estimated beta distribution is then used to transform the scales in other years (De Jonge et al., 2014). The method is successfully applied in the study of long-term 
happiness change in the Netherlands (DeJonge et al., 2016). However, as the authors themselves have noted, the limitation of this approach is that result is subject to the choice of the reference survey (De Jonge et al., 2014;De Jonge et al., 2017).

To overcome the limitations of the above-mentioned approaches, we introduce the Latent Attitude Method (hereafter LAM) to address the problem of scale incomparability. Compared with the previous approaches, our approach has two advantages. First, we utilize the full distribution of response categories instead of dichotomizing the responses. Second, our approach is reference-free. This is in contrast with the reference distribution method that covert other survey responses based a reference survey that chosen by the researcher. Our method borrows strength from two research traditions: (1) the latent variable method in attitude research (Powers and Xie, 2008), and (2) the comparable distribution condition in survey design evaluation(Couper, 2011). The latent variable approach considers individuals' attitudes to be latent, continuous constructs that underly observed discrete categories. Through reasonable assumption of the underlying distribution of the attitudes under study (normal distribution in our case), researchers can estimate the parameters that define the underlying distribution, as well as the thresholds that partition the continuous latent variable into corresponding categories.

The comparable distribution condition, put more broadly, assumes that the properties of the variables remain unchanged as long as the population under study are the same. While this condition may be straightforward, it serves as the premises for survey design evaluation in many fronts, such as testing survey mode change (Couper, 2011), or measurement change (Jaeger, 1997). For example, survey methodologists often need to conduct random experiments to evaluate the performances of different survey modes, such as web survey versus telephone survey (e.g., Fricker et al., 2005). The random assignment ensures that the properties of the variable understudy to be the same regardless 
of survey mode. In some other cases when random experiments are not available, researchers also use matching techniques to ensure that the samples are comparable from each other. A classic example is David Jaeger's work that bridges the Census Bureau's old and new measurements of educational attainment in the Current Population Survey (Jaeger, 1997). Jaeger matched individuals who answered old education questions with those who answered new one in two adjacent years. Under the condition that the distributions of the educational attainment are the same between the two groups, Jaeger created a recording metrics that convert old measurement to the new ones.

Our rationale to address cross- survey incomparability is similar to the rationale of addressing survey mode change or scale change within the same survey. In our analysis, we first model individuals' observed ordinal response using ordered probit model with unknown mean and survey-specific cutoffs. The key to identify these unknown parameters is the comparable distribution condition. Specifically, we leverage the situations when different time-series surveys overlap in certain years. When two or more surveys overlap in a given year, we assume that the same latent attitude is measured as if two measurement scales are randomly given to two independent samples drawn from the same population. Thus, we can assume the same statistical properties for the latent attitude. In so doing, we are able to reduce the number of unknowns to be less than the number of established equations and estimate the best-fit parameters with maximum likelihood method.

The rest of the paper precedes as follows. Next, we introduce the latent attitude method. We then evaluate the performances of the LAM approach with simulated data. Last, we apply the LAM method in an empirical case that investigates the long-term trend in Americans' attitude toward China from 1974 to 2019. 


\section{The Latent Attitude Method}

In this section, we introduce LAM approach. We first discuss the assumption of the model. We then move to the individual attitude model where we express individual's latent attitudes with ordered probit model. We then elaborate the comparative condition.

\subsubsection{Model assumptions}

Our approach requires three assumptions. First, the representative sample condition assumes that a survey's observations are drawn from a representative sample of the population. In another words, we assume no sampling bias specific to a given survey. Second, the time-invariant survey questionnaires assume that for any given questionnaires, the level of response scales are understood consistently by all respondents, regardless of the time of the interview. In other words, we assume the thresholds the divide latent attitudes to be only survey specific. Third, comparable distribution in the overlapping years. We assume in any given year, all surveys are measuring the same distribution of attitude in population, though the measurements could vary across surveys.

\subsection{Individual Model}

First we build individual attitude model. We assume individual $i$ 's true attitude toward a given issue in year $t$ to be an unobserved continuous variable $y_{i t}^{*}$. It can be expressed as follows:

$$
y_{i t}^{*}=\mu_{t}+\eta_{i t}, \text { where } \eta_{i t} \sim N(0,1),
$$

Function 1 shows that individuals' latent attitudes are centered around population 
mean $\mu_{t}$, and vary from individual to individual. We denote individuals' idiosyncratic error as $\eta_{i t}$ that follows standard normal distribution. Our primary goal is to identify the central tendency of population attitude, i.e. $\mu_{t}$.

Next, we move to the measurement model. Assume that we have multiple likert-type survey questionnaires that measure the same latent attitude. We index these questionnaires as $q$ where $q=1,2, . . Q$. Readers can think of $q$ to be a particular attitude questionnaire that asked repeatedly over time in large cross-sectional surveys such as GSS.

For each survey questionnaire $q$, we further denote it measures attitude with $K_{q}$ ordered response categories. In a typical probit model set - up, the measurement model can be expressed as follows :

$$
y_{i t q}= \begin{cases}1 & \text { if } y_{i t}^{*} \in\left(-\infty, \tau_{q, 1}\right] \\ 2 & \text { if } y_{i t}^{*} \in\left(\tau_{q, 1}, \tau_{q, 2}\right] \\ . . & \text { if } y_{i t}^{*} \in\left(\tau_{q, k-1}, \tau_{q, k}\right] \\ k & \text { if } y_{i t}^{*} \in\left(\tau_{q, K},+\infty\right)\end{cases}
$$

Where $y_{i t q}$ is the observed response scale that has $K_{q}$ mutually exclusive values. The assumption time-invariant survey questionnaires denotes that the thresholds parameters, $\tau$, is survey-specific, and does not change over $t$. Questionnaire $q$ defines time-invariant attitude ranges $\left[\tau_{q 0}=-\infty, \tau_{q 1}, \ldots, \tau_{q K_{q}}=+\infty\right]$ that partition continuous attitude into categorical responses. 
Combining Equ 1 and Equ 2 predicts

$$
y_{i t q}=k \Leftrightarrow \tau_{q, k-1}-\mu_{t}<\eta_{i t} \leq \tau_{q, k}-\mu_{t} .
$$

Equation 3 suggest that the individual's idiosyncrasies can be expressed as differences between the threshold and the sample mean.

Now we are able to write down the probability of random event of any individual $i$ responding $y_{i t q}=k$ in the form of $\eta_{i t}$,

$$
\begin{aligned}
\operatorname{Pr}\left(y_{i t q}=k\right) & =\operatorname{Pr}\left(\tau_{q, k-1}-\mu_{t}<\eta_{i t} \leq \tau_{q, k}-\mu_{t}\right) \\
& =\Phi\left(\tau_{q, k}-\mu_{t}\right)-\Phi\left(\tau_{q, k-1}-\mu_{t}\right)
\end{aligned}
$$

where $\Phi(z)$ is the cumulative density functions of Gaussian distribution.

\subsection{Estimation}

The unknown parameters $\tau, \mu, \eta$ are estimated by maximizing the joint likelihood $L$,

$$
\begin{aligned}
L & =\prod_{q} \prod_{t \in\left[1, l_{q}\right]} \prod_{k \in\left[1, K_{q}\right]} \operatorname{Pr}(k)^{N_{q t k}} \\
& =\prod_{q} \prod_{t \in\left[1, l_{q}\right]} \prod_{k \in\left[1, K_{q}\right]}\left(\Phi\left(\tau_{q, k}-\mu_{t}\right)-\Phi\left(\tau_{q, k-1}-\mu_{t}\right)\right)^{N_{q t k}},
\end{aligned}
$$

where $N_{q t k}=\sum_{i} I\left(y_{i t q}=k\right)$ is the number of individuals responding $k$ to questionnaire $q$ in year $t$.

Note that $L$ is translation invariant and Equation 4 has an infinite number of optimal 
solutions. Say there is a solution $(\hat{\tau}, \hat{\mu}, \hat{\eta})$ at the maximal $L$, i.e.,

$$
L(\hat{\tau}, \hat{\mu}, \hat{\eta}) \geq L(\tau, \mu, \eta), \quad \forall \tau, \mu, \eta
$$

It is easy to construct another solution by shifting all parameters with any given constant $c$, that is equally good as $(\hat{\tau}, \hat{\mu}, \hat{\eta})$, i.e.,

$$
L(\hat{\tau}, \hat{\mu}, \hat{\eta})=L(\hat{\tau}+c, \hat{\mu}+c, \hat{\eta}+c) .
$$

Therefore we need to manually specify the value of one parameter in $(\hat{\tau}, \hat{\mu}, \hat{\eta})$ to avoid infinitely parallel solutions. Without loss of generality, in practice $\mu$ of the first year is set to 0 , which will not affect the estimated trend except a constant shift. Our estimation turns out a constrained optimization problem as follows,

$$
\begin{array}{ll}
\max _{\tau, \mu, \eta} & \prod_{q} \prod_{t \in\left[1, l_{q}\right]} \prod_{k \in\left[1, K_{q}\right]}\left(\Phi\left(\tau_{q, k}-\mu_{t}\right)-\Phi\left(\tau_{q, k-1}-\mu_{t}\right)\right)^{N_{q t k}} \\
\text { s.t. } & \mu_{0}=0
\end{array}
$$

We set the attitude of the earliest year to be zero. To facilitate the optimization process, we feed the model with the initial values that draw from a simpler orderedprobit model that ignore the cross-survey scale differences.

\subsection{Use overlapping years to anchor multiple surveys}

A key to solving Equ 5 with multiple surveys relies the comparable distribution condition, i.e., $\mu_{t}$ does not vary across surveys,

$$
\mu_{t, q=1}=\mu_{t, q=2}=\cdots=\mu_{t}
$$


In fact, $\mu_{t}$ propagates the information between surveys to properly anchor their thresholds $\tau$.

Proposition 2.1 Two surveys can be anchored only when they have at least one overlapping year.

The proof is obvious. If two surveys $q$ and $q^{\prime}$ does not overlap in any year, Equ 4 can be split into two terms, covering the years of survey $q$ and the years of survey $q^{\prime}$ respectively. Setting $\mu_{0}=0$ (without loss of generality let us assume $q$ covers the first year) does not help exclude infinite maxima of the term of the years of $q^{\prime}$, and there would be an infinite number of equally good solutions differing only a constant in all parameters of $q^{\prime}$. To overcome this issue, we need to further manually specify the value of one parameter in $(\hat{\tau}, \hat{\mu}, \hat{\eta})$ related with $q^{\prime}$. For example, we may choose to specify any $\mu$ in the years of $q^{\prime}$. However, the arbitrariness in selecting such a parameter and its value makes it unreliable to comapre the values of $\mu$ of survey $q$ and those of survey $q^{\prime}$.

Next we introduce the requirement of connected surveys to anchor multiple surveys. Starting with a collections of distinct surveys, we construct an undirected network of surveys by connect every pair of surveys if they overlap for at least one year. Our model works for a subset of surveys in which any two surveys are directly or indirectly connected, i.e., a path exists on this network between them (Fig 1).

[Figure 1 inserts here]

For a group of connected surveys, it is sufficient to specify the value of only one parameter (e.g., $\mu_{0}$ ) to train on all survey observations and produce comparable estimates of yearly attitudes. 


\section{Simulation Study}

Before applying the LAM method to the real-world data, we assess the performance of the method with simulated data.

\subsection{Simulation Design}

We first simulate the attitudes distribution of the population under study. Specifically, we generate 10 years of data with 100,000 case each year. Each year, individuals' latent attitude $y_{i t}^{*}$ are drawn from a normal distribution with a random mean. The simulated latent attitude distributions are displayed in panel a of Figure 2.

Next, we simulate two surveys that measures the latent attitudes with two different sets of ordinal scales. Both surveys draw random samples from the population each year, thus they both considered to be representative from the population. The first survey draws $20 \%$ sample from the population, and runs from year 1 to year 6 . It measures latent attitude with a 1- 4 scale. The second survey draws $10 \%$ sample from the population, and runs from year 5 - year 10 . It measures latent attitude in a 1- 5 scale. These ordinal scales are assigned by setting-up survey- specific threshold values $\tau$ s, such that the relationships between the latent value, $y_{i t}^{*}$ and $\tau$ s follow equation 2 . We assign survey - specific threshold values, indicated in the column 2 and 3 of Table 1

Panel $\mathrm{b}$ of Figure 2 shows the cumulative distributions of the ordinal responses of the two surveys.

[Figure 2 Inserts Here]

\subsection{Simulation Results}

Panel c in Figure 2 shows the LAM estimates with the bootstrapped $95 \%$ confidence interval. Two findings are worth noticing. First, the LAM estimates are very close to 
the actual mean of attitudes, suggesting the validity of the method. Second, sample size affects the estimation precision. As indicated by the confidence intervals, the LAM estimations achieve the highest precision in the years when the two surveys overlap (year 5 and 6 ). This is because the sample size in year 5 and 6 are the largest in pooled data.

The estimated thresholds using LAM approach are presented in Table 1. The table show that differences between the actual and estimated thresholds are very small.

[Table 1 Inserts Here]

\section{Empirical Example : Attitudes Toward China}

We demonstrate the utility of the LAM approach with an empirical example that analyzes general public's' attitude toward the People's Republic of China in the United States. The U.S. - China relationship is one of the most important bilateral relationship across globe. The questions about attitude toward China are routinely asked in large social surveys (e.g. GSS) and multiple polling organizations (e.g. PEW and Gallup).

How do the general public's' attitude toward China evolve over time? We attempt to address this question with pool times series data. We draw data from three large survey archives, the Roper Center for Public Opinion Research (ROPER), the National Opinion Research Center (NORC), and Inter-university Consortium for Political and Social Research (ICPSR). We selected questionnaires on public attitude toward China from these three survey archives based on three criteria: (1) The questionnaires must measure general attitude toward China. We excluded questionnaires about specific attitude toward Chinese people, government etc.;(2) The targeted population is the entire U.S. population. We excluded regional surveys;(3) The same questionnaires are asked at least in two different years. We excluded surveys that conducted only one year. We only included response choices that asked in the survey, thus don't knows and refused to answer are not included in the final data set. 
The final dataset contains information from 101 cross-sectional public opinion surveys, administered by 10 organizations, spanned between $1974-2019$. The questionnaires in the final dataset are drawn from wide ranges of sources, including national social surveys (e.g. General Social Survey), polls conducted by public opinion research companies (e.g. Gallup and Pew), and television network polls (e.g. ABC and CBS). We constructed 13 time-series variables based on the survey organizations, question wording and response categories. Questionnaires surveyed in different years are categorized as the same series if they are administered by the same organization, have the exact wording and response categories. As a result, questionnaires that administered by the same survey organization but have different response category are classified as different series (such as GALLUP_4 and GALLUP_10 in Table 2)

Table 2 presents the detailed description of the data. It shows that the wordings of these questionnaires are almost identical, and this guarantees that they all measure the concept of latent attitude toward China. However, the response categories vary from a simple binary scale indicating favorable or unfavorable attitude, to a 0 - 100 warmth scale. The most common response scale is four, where the respondents rate their attitude toward China as very favorable, somewhat favorable, somewhat unfavorable, or very unfavorable. The longest running question series was administered by Gallup, which asked respondents attitude toward China with a four-scale response (GALLUP_4).

\section{[Table 2 Inserts Here]}

Figure 3 shows the percentages of favorable attitudes toward China. Overall, there appear to have a decline on the percentages of Americans who are in favor of China since 1990. Prior to 1990, most polls found that the percentages Americans who are in favor of China were well above 40. The favorable attitudes were as high as over 80 percent in a 1989 Gallup poll. Americans' attitude toward China experienced experienced drastic decline since 1990 and remain low (40 percent or less) since then. Figure 3 also show 
discrepancies across surveys. Questionnaires from several organizations, such as CBS and Growth from Knowledge Networks (USKN_5), show persistent lower percentages of favorable attitudes toward China compared to the questionnaire conducted by other survey organizations.

\section{[Figure 3 Inserts Here]}

Figure 4 shows the estimated attitude toward China with LAM approach. Compared with the survey-specific trends in Figure 3, the LAM estimates provide a clearer picture of the attitudinal change over time. Overall, Americans' attitude toward China largely corresponds with the U.S. - China relationships. For example, the 1970s was a period of rapprochement, during which time United Sates formally established diplomatic relationship with the People's Republic of China. As a result, the public opinion of China kept rising throughout the 1970s. Americans' relatively favorable attitude toward China also persisted throughout the 1980s. In 1989, however, the Chinese government cracked down student-led protests that calling for democracy in the Tiananmen Square, and this led a devastating effect of American's attitude toward China. After 1989, Americans' attitudes never reached to a point that comparable to the time in the 1970s. There are nevertheless noticeable attitudinal changes in the 1990s and 2000s. For example, U.S.China relationships improved after the September 11 terrorist attacks (Wang, 2009), and this led to an increase in Americans' attitude toward China. In the current Trump administration, the U.S. - China relationship experienced rapid deterioration, and this is reflected to the declining attitude toward China since 2018.

[Figure 4 Inserts Here] 


\section{Conclusion and Discussion}

The use of survey data to understand societal attitudes has long enthralled social scientists. With the accumulation of survey data, there is a growing interest to pool different sources of survey to study trends of social attitudes. However, the use of pooled data is handicapped by one measurement issue: different surveys may adopt different instruments therefore the results among surveys are not strictly comparable. We develop Latent Attitude Method (LAM) to address the issue of survey scale incomparability in the study of attitudinal change. The core of our method is that, when two or more surveys overlap in a given year, we assume that the same latent attitude is measured as if two measurement scales are randomly given to two independent samples drawn from the same population. With this condition, we are able to estimate unknown cut-offs and year-specific mean levels using maximum likelihood method.

Our method can be fruitfully applied to the contexts where multiple surveys measure the same latent constructs. We have shown that the method can be used to understand Americans' changing attitudes toward China. The methods can also be applied to other contexts when scholars are interested in the long -term change of subjective well-being, such as happiness or self-rated health. Besides trend analysis, the method can also be used in the case of geographical variations of attitudes or subjective well-being. Recall that the key to identify the unknown cut-off point and the mean in the trend analysis is the overlapping year assumption. We suggest that in the case of the geographic variations, the same rationale can be applied if two or more surveys overlap at the same geographic unit. If, for example, two surveys overlapped in the same geographic unit, we can consider the two surveys measure the same latent attitudes, and anchor the survey based on the overlapping geographic location.

The current method can be extended in the following way. We assume the surveys are 
free from sampling errors. However, this assumption is unlikely hold in real world. There are at least two types of errors to be concerned about: the survey series- specific error and year-specific error. The survey-series specific errors refer to the errors associated with a particular survey series. For example, some surveys may be consistently over or under sample certain demographic groups or geographic locations across all waves. The year-specific errors refer to the cases when all surveys are subject to the same amount of errors in a given year. However, because we do not know the ground truth, we suggest that this issue can be tackled if additional information of the surveys are available. For example, if the distributions of respondents' demographics are known, researchers can address potential survey series specific errors by comparing respondents demographics and re-weight the series to make it nationally representative. 


\section{References}

Thornton, A. (1985). Changing Attitudes Toward Separation and Divorce: Causes and Consequences. American Journal of Sociology, 90(4), 856-872. https://doi.org/ $10.1086 / 228147$

Steeh, C., \& Schuman, H. (1992). Young White Adults: Did Racial Attitiudes Change in the 1980s? American Journal of Sociology, 98(2), 340-367. https://doi.org/ $10.1086 / 230011$

DiMaggio, P., Evans, J., \& Bryson, B. (1996). Have Americans' Social Attitudes become More Polarized? American Journal of Sociology, 102(3), 690-755. https:// doi . $\operatorname{org} / 10.1086 / 230995$

Scott, J., Alwin, D. F., \& Braun, M. (1996). Generational Changes in gender-role attitudes: Britain in cross-national perspective. Scoiology, 30(3), 471-492.

Jaeger, D. A. (1997). Reconciling the old and new census bureau education questions: Recommendations for researchers. Journal of Business and Economic Statistics, 15(3), 300-309. https://doi.org/10.1080/07350015.1997.10524708

Fricker, S., Galesic, M., Tourangeau, R., \& Yan, T. (2005). An experimental comparison of web and telephone surveys. Public Opinion Quarterly, 69 (3), 370-392. https: //doi.org/10.1093/poq/nfi027

Jackman, S. (2005). Pooling the polls over an election campaign. Australian Journal of Political Science 40(4), 499-517. https://doi.org/10.1080/10361140500302472

Osberg, L., \& Smeeding, T. (2006). FAIR INEQUALITY? ATTITUDES TOWARD PAY DIFFERENTIALS: THE UNITED STATES IN COMPARATIVE PERSPECTIVE. American Sociological Review, 71(6), 450-473. https://doi.org/10. $4324 / 9780203881972$ 
Wang, C. (2009). George W. Bush and China: Policies, Problems, and Partnerships. Lexington Books.

Cotter, D., Hermsen, J. M., \& Vanneman, R. (2011). The end of the gender revolution? gender role attitudes from 1977 to 2008. American Journal of Sociology, 117(1), 259-289. https://doi.org/10.1086/658853

Couper, M. P. (2011). The future of modes of data collection. Public Opinion Quarterly, 75(5 SPEC. ISSUE), 889-908. https://doi.org/10.1093/poq/nfr046

Semyonov, M., Raijman, R., \& Gorodzeisky, A. (2011). The Rise of Anti-foreigner Sentiment in European Societies, 1988 - 2000. American Sociological Review, 1(2004), $426-449$.

De Jonge, T., Veenhoven, R., \& Arends, L. (2014). Homogenizing Responses to Different Survey Questions on the Same Topic: Proposal of a Scale Homogenization Method Using a Reference Distribution. Social Indicators Research, 117(1), 275300. https://doi.org/10.1007/s11205-013-0335-6

DeJonge, T., Veenhoven, R., Kalmijn, W., \& Arends, L. (2016). Pooling Time Series Based on Slightly Different Questions About the Same Topic Forty Years of Survey Research on Happiness and Life Satisfaction in The Netherlands. Social Indicators Research, 126(2), 863-891. https://doi.org/10.1007/s11205-015-0898-5

De Jonge, T., Veenhoven, R., \& Kalmijn, W. (2017). Diversity in Survey Questions on the Same Topic (Vol. 68). Cham, Springer International Publishing. https://doi.org/10.1007/978-

\section{3-319-53261-5}

Stimson, J. A. (2018). Public opinion in America: Moods, cycles, and swings, second edition. Taylor; Francis. https://doi.org/10.4324/9780429494796 


\section{$6 \quad$ Tables and Figures}

Table 1: Actual and Estimated Thresholds

\begin{tabular}{lllll}
\hline Thresholds & $\begin{array}{l}\text { Survey 1 } \\
\text { (True Value) }\end{array}$ & $\begin{array}{l}\text { Survey 2 } \\
\text { (True Value) }\end{array}$ & $\begin{array}{l}\text { Survey 1 } \\
\text { (Estimates) }\end{array}$ & $\begin{array}{l}\text { Survey 2 } \\
\text { (Estimates) }\end{array}$ \\
\hline$\tau_{1}$ & -1.577 & -2.206 & -1.554 & -2.229 \\
$\tau_{2}$ & 0.020 & -0.824 & 0.063 & -0.808 \\
$\tau_{3}$ & 1.604 & 0.867 & 1.670 & 0.892 \\
$\tau_{4}$ & $\mathrm{NA}$ & 1.404 & $\mathrm{NA}$ & 1.445 \\
\hline
\end{tabular}


Table 2: Data on Attitudes Toward China

Time

series

variable

Question Wording

Scale Organization

Total

variable

\begin{tabular}{ll}
\hline ABC & $\begin{array}{l}\text { Would you say you generally have a favorable or unfavorable } \\
\text { impression of China? }\end{array}$ \\
\hline CBS_3 & $\begin{array}{l}\text { Are your feelings toward China generally favorable, generally } \\
\text { unfavorable, or neutral? }\end{array}$
\end{tabular}

How about the following countries, please tell me if you have

TRA_4 a very favorable, somewhat favorable, somewhat unfavorable or very unfavorable opinion of...China.

\begin{tabular}{llll}
\hline PEW & $\begin{array}{l}\text { Do you have a very favorable, mostly favorable, mostly } \\
\text { unfavorable,or very unfavorable attitude toward China? }\end{array}$ & 4 & PEW Research Center \\
\hline & Is your overall opinion of each of the following countries very
\end{tabular}

Is your overall opinion of each of the following countries very
ORC_4
favorable, mostly favorable, mostly unfavorable, or very
unfavorable? China

\begin{tabular}{|c|c|c|c|c|}
\hline PSRA_4 & $\begin{array}{l}\text { Is your overall opinion of China very favorable, mostly } \\
\text { favorable, mostly unfavorable, or very unfavorable? }\end{array}$ & 4 & $\begin{array}{l}\text { Princeton Survey } \\
\text { Research Associates }\end{array}$ & 3 \\
\hline
\end{tabular}

\begin{tabular}{llll}
\hline ZOGBY & How would you describe your impression of China? Is it very & & Zogby International \\
-4 & favorable, somewhat favorable, somewhat unfavorable, or & 4 & very unfavorable?
\end{tabular}

\begin{tabular}{ll}
\hline GALLUP & $\begin{array}{l}\text { Is your opinion of China very favorable, mostly favorable, } \\
\text { mostly unfavorable or very unfavorable? }\end{array}$ \\
\hline I'd like you to rate your feelings toward some countries,
\end{tabular}

$\begin{array}{ll} & \text { institutions and people, with } 100 \text { meaning a very warm, } \\ \text { TRA_5* } & \text { favorable feeling and } 0 \text { meaning a very cold, unfavorable }\end{array}$ feeling, and 50 meaning not particularly cold or warm:

$5 \quad$ TNS Opinion and 4 China

Please rate your feelings towards some countries, with 100 meaning a very favorable feeling, 0 meaning a very unfavorable USKN_5* feeling, and 50 meaning a feeling that is neither favorable nor
unfavorable. You can use any number between 0 and 100, $5 \quad$ GfK Knowledge Networks the higher the number, the more favorable your feelings toward this country: China

You notice that the ten boxes on this card go from the highest position of plus five-for something you have a very favorable

GALLUP opinion of-all the way down to the lower position of minus five

_10 - for something you have a very unfavorable opinion of.

$10 \quad$ Gallup

Please tell me how far up the scale or how far down the scale you rate this nation: China.

You will notice that the boxes on this card go from the highest position of "plus 5 " for a country which you like very much to

GSS the lowest position of "minus 5" for a country you dislike very much. How far up the scale or how far down the scale would $10 \quad$ National Opinion Research Center you rate the following countries? F.China

*The 0 - 100 warmth scale was reported as 1-5 scale in the Roper Center Archive 
Fig. 1: Connected surveys. (a) Five surveys are running in some years (grey in the left panel). (b) Surveys A and B are directly connected as they overlap in 2001, B and C are directly connected with an overlapping year 2002. Surveys $\mathrm{A}$ and $\mathrm{C}$ are indirectly connected as there is a path between them. Surveys A, $\mathrm{B}, \mathrm{C}$ are not connected to survey D or E.

(a)

\begin{tabular}{|l|l|l|l|l|l|l|l|}
\hline & 2000 & 2001 & 2002 & 2003 & 2004 & 2005 & 2006 \\
\hline Survey A & & & & & & & \\
\hline Survey B & & & & & & & \\
\hline Survey C & & & & & & & \\
\hline Survey D & & & & & & & \\
\hline Survey E & & & & & & & \\
\hline
\end{tabular}

(b)

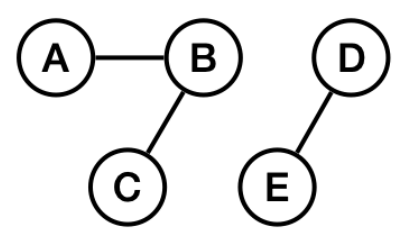


Fig. 2: Simulated Data

(a) Latent Attitude Distribution

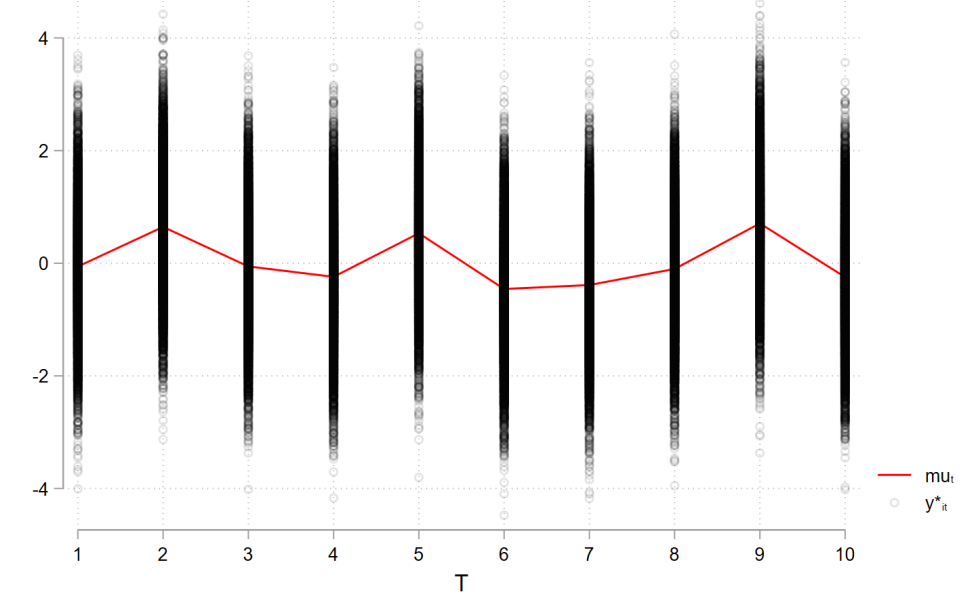

(c) LAM Estimates

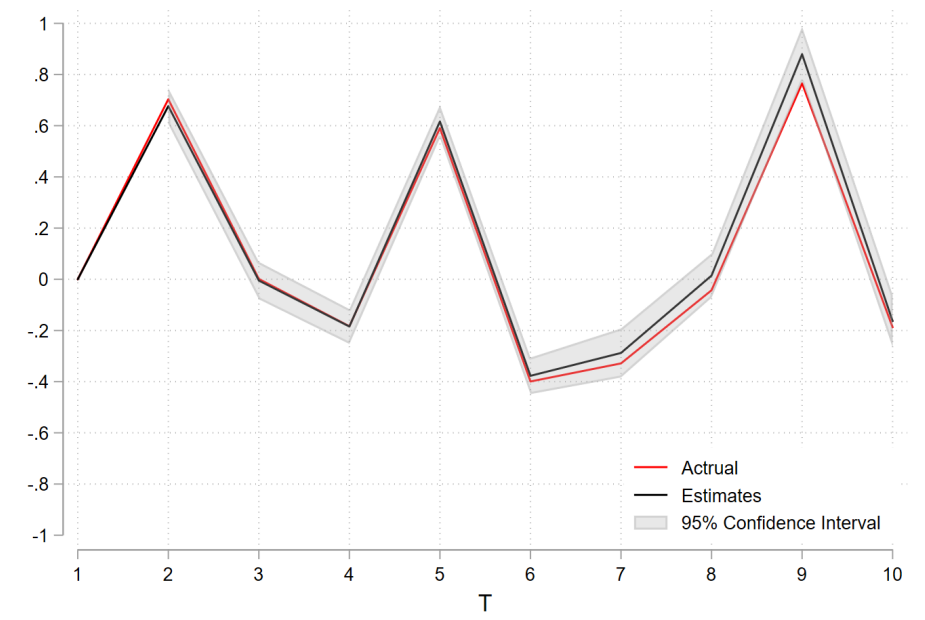

(b) Cumulative distribution of ordinal scales from two simulated surveys

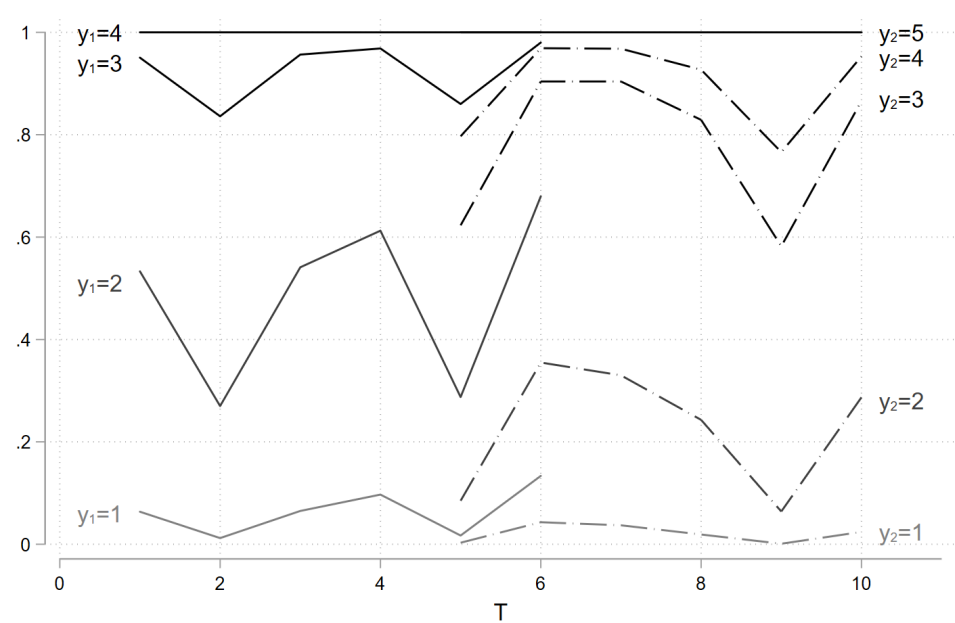


Fig. 3: Percentage favorable attitude toward China

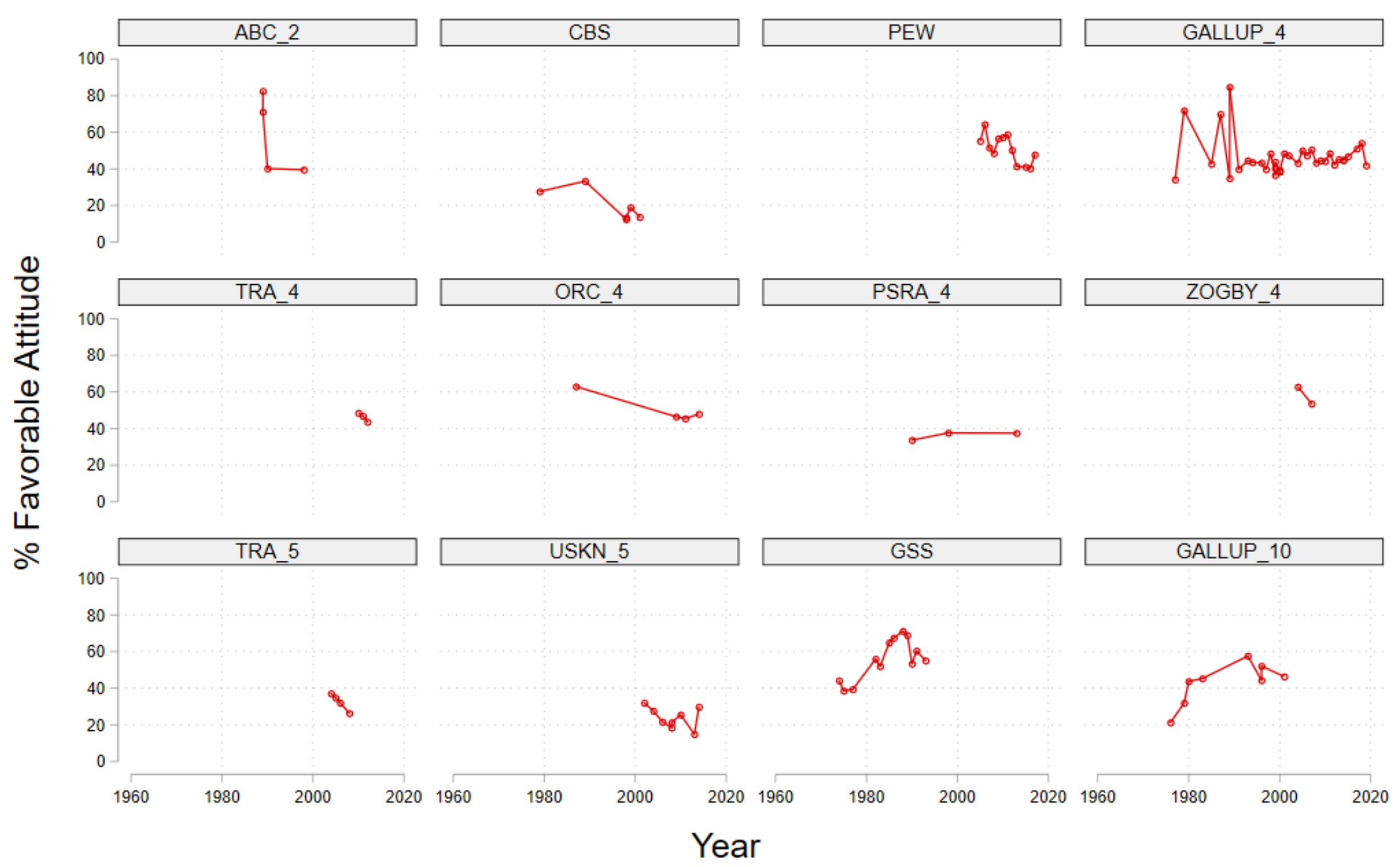


Fig. 4: Latent Attitude Method Estimation on Attitude Toward China

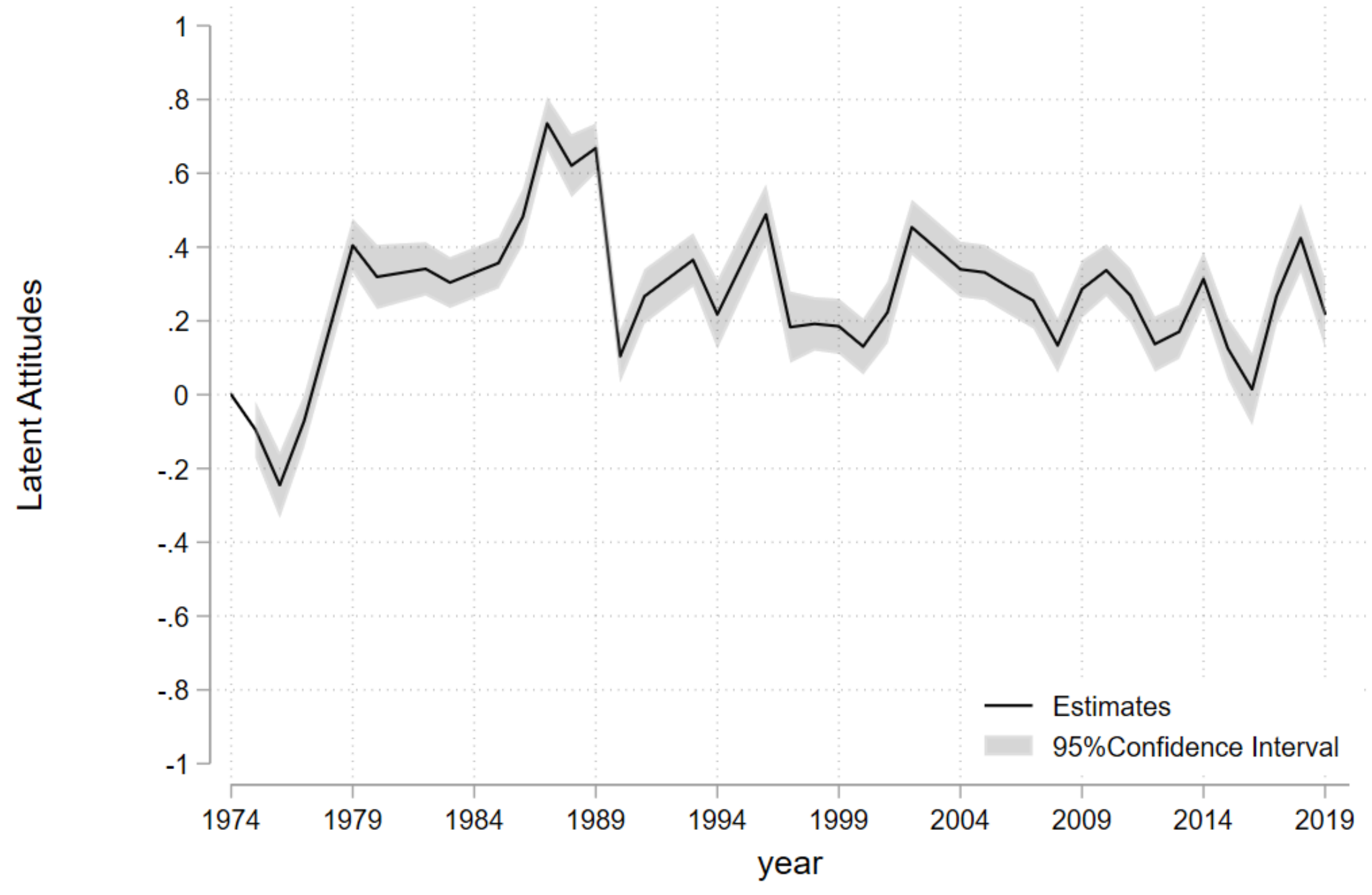

